\title{
Use of the Last Menstrual Period (LMP) in Timing Human Pregnancies
}

\section{To the Editor}

A common, but often attacked, method of dating human pregnancy is to use the first day of the last normal menstrual period (LMP). This method has repeatedly been shown to be valid when dealing with large numbers of pregnancies, i.e., in situations in which the accurate determination of the age of an individual fetus is not the critical issue. Implicit in the LMP dating method are the assumptions that 1) ovulation-fertilization takes place 14 days after the LMP and 2) the woman's recall of the LMP is accurate. The first assumption is known to be invalid because the interval between the LMP and ovulation is quite variable, with only a mean of 14 days.

The woman's recall of the LMP is also open to question. She may not be sure of the LMP or she may have difficulty with the term "normal." Another type of inaccuracy was reported by Frazier ('59), who reported data on the stated LMP from 1,727 pregnancies. $\mathrm{He}$ noted a bias for the selection of certain dates, particularly the 10th, 15th, 20th, and 25th. Certain of the subgroups he studied also had a propensity to name the 27-28th.

Of 635 fetuses that I have studied over the past 8 years, a specific date for the LMP was given in 365 instances. LMPs starting on the 10th, 20th, 25th, 27th, and 28th were significantly $(\mathrm{P}<0.05)$ more common than predicted and LMPs starting on the 9th and 11th were significantly $(\mathrm{P}<0.05)$ less common. For comparative purposes, the dates of delivery of these fetuses were analyzed. The overall pattern did not differ significantly from the theoretic distribution and no particular date occurred with a significant excess or deficiency.
The distribution of the overreported dates for the LMP suggests that women, in many cases, estimate the LMP. To test this hypothesis, I made an informal survey of women around the hospital (employees, patients, parents). Each woman was asked to name a date (number) in response to one of several questions. In most cases, the way the question was phrased was associated with a statistically significant propensity for the woman to name a particular number. The key phrases and the numbers selected were: first week (3), second week (10), third week (21), fourth week (28), middle of the month (15), early in the month (3), in the early part of the month $(3,4,5)$, in the first part of the month (5), in the beginning of the month $(4,5)$, near the end of the month (29), in the late part of the month $(21,29)$, in the latter part of the month $(27,30)$, in the last week $(29,30)$, and late in the month (28).

While this survey (for which I gained the reputation as a nut) does not identify the underlying bias that leads women to name certain dates for the LMP, it does suggest that the bias could be related to the way the date is elicited. Thus, while the use of the LMP to estimate fetal age is still of general use, it should be treated with liberal skepticism and be regarded as only a very rough estimation.

\section{LITERATURE CITED}

Frazier, T.M. (1959) Error in reported date of last menstrual period. Am. J. Obstet. Gynecol., 77:915-918.

Mason Barr, JR. Department of Pediatrics University of Michigan Ann Arbor, Michigan 48109 